\title{
metal-organic compounds
}

Acta Crystallographica Section E

\section{Structure Reports}

Online

ISSN 1600-5368

\section{Tetraaquabis(5-hydroxynicotinato- $\kappa(N)$ - cadmium(II)}

\section{Mei-Xiang Jiang and Yun-Long Feng*}

Zhejiang Key Laboratory for Reactive Chemistry on Solid Surfaces, Institute of Physical Chemistry, Zhejiang Normal University, Jinhua, Zhejiang 321004, People's Republic of China

Correspondence e-mail: sky37@zjnu.edu.cn

Received 22 October 2008; accepted 1 November 2008

Key indicators: single-crystal X-ray study; $T=296 \mathrm{~K}$; mean $\sigma(\mathrm{C}-\mathrm{C})=0.002 \AA$; $R$ factor $=0.016 ; w R$ factor $=0.042 ;$ data-to-parameter ratio $=13.4$.

The title compound, $\left[\mathrm{Cd}\left(\mathrm{C}_{6} \mathrm{H}_{4} \mathrm{NO}_{3}\right)_{2}\left(\mathrm{H}_{2} \mathrm{O}\right)_{4}\right]$, was obtained by the reaction of cadmium chloride with 5-hydroxynicotinic acid. The $\mathrm{Cd}^{\mathrm{II}}$ atom is located on an inversion centre and is coordinated by two $\mathrm{N}$ atoms from two 5-hydroxynicotinic acid ligands and four water molecules in a distorted octahedral geometry. The structure is stabilized by intermolecular $\mathrm{O}-$ $\mathrm{H}$... O hydrogen bonds, forming a three-dimensional network.

\section{Related literature}

For cadmium componds and their photoluminescent properties, see: He et al. (2008); Kang et al. (2007); Zhang et al. (2006); Zora et al. (2006).

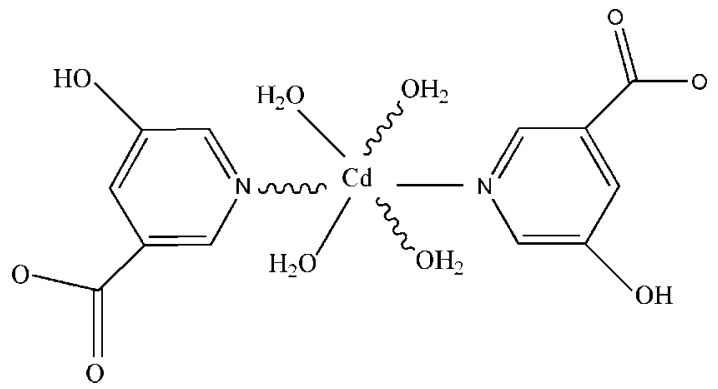

\section{Experimental}

Crystal data

$\left[\mathrm{Cd}\left(\mathrm{C}_{6} \mathrm{H}_{4} \mathrm{NO}_{3}\right)_{2}\left(\mathrm{H}_{2} \mathrm{O}\right)_{4}\right]$

$M_{r}=460.68$

Triclinic, $P \overline{1}$

$a=7.2190(1) \AA$ $b=7.2510(1) \AA$

$c=8.9260(1) \AA$

$\alpha=70.377(1)^{\circ}$

$\beta=68.154$ (1)

$\gamma=65.7170(10)^{\circ}$

$V=385.97(1) \AA^{3}$

Data collection

Bruker APEXII diffractometer Absorption correction: multi-scan (SADABS; Sheldrick, 1996)

$T_{\min }=0.667, T_{\max }=0.903$

$Z=1$

Mo $K \alpha$ radiation

$\mu=1.48 \mathrm{~mm}^{-1}$

$T=296(2) \mathrm{K}$

$0.27 \times 0.17 \times 0.07 \mathrm{~mm}$

Refinement

$R\left[F^{2}>2 \sigma\left(F^{2}\right)\right]=0.016$

$w R\left(F^{2}\right)=0.042$

$S=1.09$

1759 reflections

131 parameters

7 restraints

6067 measured reflections 1759 independent reflections 1754 reflections with $I>2 \sigma(I)$ $R_{\text {int }}=0.017$

$\mathrm{H}$ atoms treated by a mixture of independent and constrained refinement

$\Delta \rho_{\max }=0.36$ e $\AA^{-3}$

$\Delta \rho_{\min }=-0.34{\mathrm{e} \AA^{-3}}^{-3}$

Table 1

Hydrogen-bond geometry $\left(\AA{ }^{\circ}\right)$.

\begin{tabular}{lcccc}
\hline$D-\mathrm{H} \cdots A$ & $D-\mathrm{H}$ & $\mathrm{H} \cdots A$ & $D \cdots A$ & $D-\mathrm{H} \cdots A$ \\
\hline $\mathrm{O} 1 W-\mathrm{H} 1 W A \cdots \mathrm{O} 2^{\mathrm{i}}$ & $0.81(2)$ & $1.94(2)$ & $2.742(2)$ & $171(3)$ \\
$\mathrm{O}^{\mathrm{ii}} W-\mathrm{H} 1 W B \cdots 3^{\text {ii }}$ & $0.79(2)$ & $2.20(2)$ & $2.973(2)$ & $164(3)$ \\
$\mathrm{O} 2 W-\mathrm{H} 2 W A \cdots \mathrm{O} 1^{\text {iii }}$ & $0.82(2)$ & $1.87(2)$ & $2.656(2)$ & $160(3)$ \\
$\mathrm{O} 2 W-\mathrm{H} 2 W B \cdots \mathrm{O} 2^{\text {iv }}$ & $0.82(2)$ & $1.93(2)$ & $2.735(2)$ & $165(3)$ \\
$\mathrm{O} 3-\mathrm{H} 3 \cdots \mathrm{O} 1^{\mathrm{v}}$ & $0.83(2)$ & $1.88(2)$ & $2.664(2)$ & $157(3)$ \\
\hline Symmetry codes: (i) & $-x+1,-y+1,-z+1 ;$ & (ii) & $-x,-y+1,-z+2 ;$ & (iii) \\
$-x+2,-y+1,-z+1 ;$ (iv) $x, y-1, z+1 ;(\mathrm{v}) x-1, y, z$. &
\end{tabular}

Data collection: SMART (Bruker, 2004); cell refinement: SAINT (Bruker, 2004); data reduction: XPREP (Bruker, 2004); program(s) used to solve structure: SHELXTL (Sheldrick, 2008); program(s) used to refine structure: SHELXTL; molecular graphics: SHELXTL; software used to prepare material for publication: SHELXTL.

Supplementary data and figures for this paper are available from the IUCr electronic archives (Reference: AT2660).

\section{References}

Bruker (2004). SAINT, SMART and XPREP. Bruker AXS Inc., Madison, Wisconsin, USA.

He, Y. H., Feng, Y. L., Lan, Y. Z. \& Wen, Y. H. (2008). Cryst. Growth Des. 8, 3586-3594.

Kang, Y., Zhang, J., Qin, Y. Y., Li, Z. J. \& Yao, Y. G. (2007). J. Mol. Struct. 784, 98-108.

Sheldrick, G. M. (1996). SADABS. University of Göttingen, Germany.

Sheldrick, G. M. (2008). Acta Cryst. A64, 112-122.

Zhang, L. Y., Zhang, J. P., Lin, Y. Y. \& Chen, X. M. (2006). Cryst. Growth Des. 6, 1684-1689.

Zora, P., Gordana, P., Marijana, V. \& Drazen, V. T. (2006). Polyhedron, 25, 2353-2362. 


\section{supporting information}

Acta Cryst. (2008). E64, m1517 [doi:10.1107/S1600536808035903]

\section{Tetraaquabis(5-hydroxynicotinato- $\kappa \mathrm{N})$ cadmium(II)}

\section{Mei-Xiang Jiang and Yun-Long Feng}

\section{S1. Comment}

There is intense research on the synthesis of the cadmium metal compounds for their interesting photoluminescent properties. A large number of these compounds have been synthesized (He et al., 2008; Zora et al., 2006; Kang et al.,2007; Zhang et al., 2006).

As illustrated in Fig. 1, the $\mathrm{Cd}$ (II) atom is coordinated by two nitrogen atoms from two 5-hydroxynicotinic acid ligands and four water molecules. Four coordinated atoms of O1W, O2W, O1WA and O2WA constitute the base of the octahedral, whereas N1 and N1A atoms occupy the apical position. The intermolecular hydrogen bonds play an important role in the formation of the three-dimensional network. As shown in Fig. 2, the intermolecular $\mathrm{O}-\mathrm{H} \cdots \mathrm{O}$ hydrogen bonds link the neighboring molecules to a three-dimensional network.

\section{S2. Experimental}

A mixture of $0.5 \mathrm{mmol} 5$-hydroxynicotinic acid and $0.5 \mathrm{mmol}$ of cadmium chloride in $10 \mathrm{ml}$ distilled water was stirred for $30 \mathrm{~min}$ at $323 \mathrm{~K}$, then the reaction mixture was filtered and well shaped colourless crystals of the title compound was obtained from the mother liquor by slow evaporation at room temperature for several days.

\section{S3. Refinement}

The $\mathrm{H}$ atoms bonded to $\mathrm{C}$ atoms were positioned geometrically [aromatic $\mathrm{C}-\mathrm{H}=0.93 \AA$ and aliphatic $\mathrm{C}-\mathrm{H}=0.97 \AA$, $\left.U_{\text {iso }}(\mathrm{H})=1.2 U_{\text {eq }}(\mathrm{C})\right]$. The $\mathrm{H}$ atoms bonded to $\mathrm{O}$ atoms were located in a difference Fourier maps and refined with $\mathrm{O}-\mathrm{H}$ distance restraints of 0.85 and $U_{\mathrm{iso}}(\mathrm{H})=1.5 U_{\mathrm{eq}}(\mathrm{O})$. 


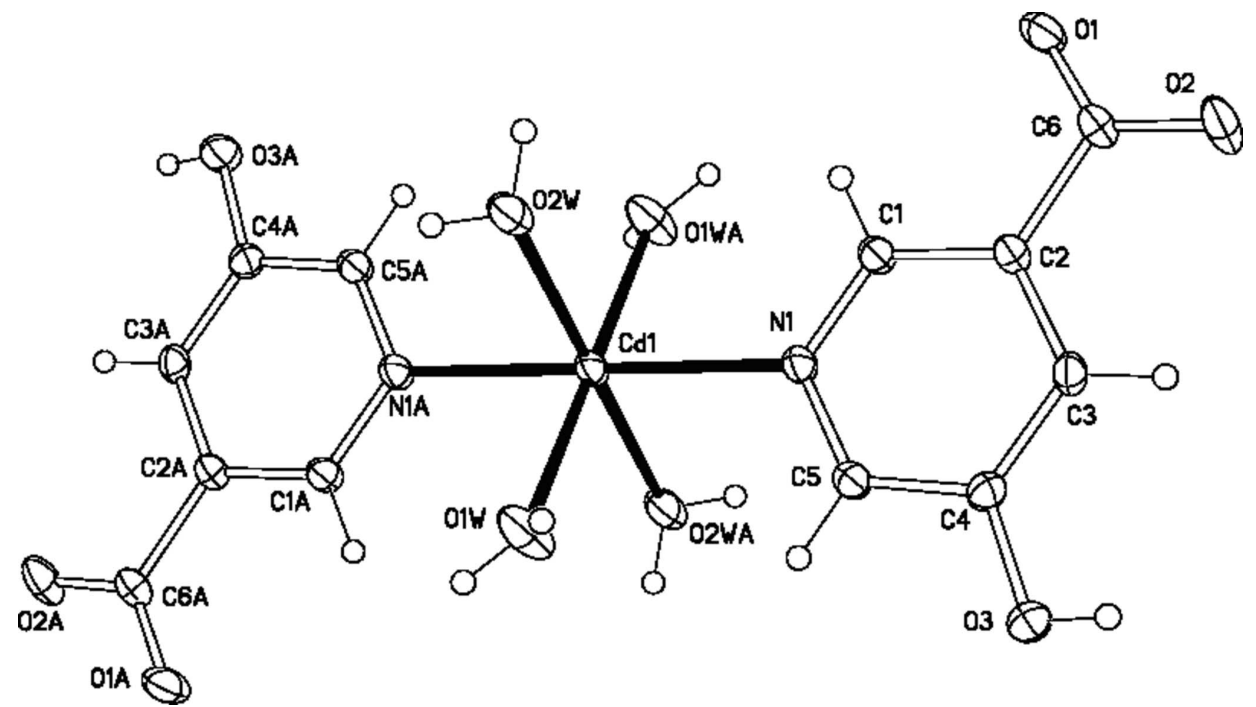

\section{Figure 1}

A view of the molecule of (I), showing the atom-labelling scheme, displacement ellipsoids are shown at the $30 \%$ probability level. [Symmetry code: (A) $-x+1,-y+1,-z+2]$.

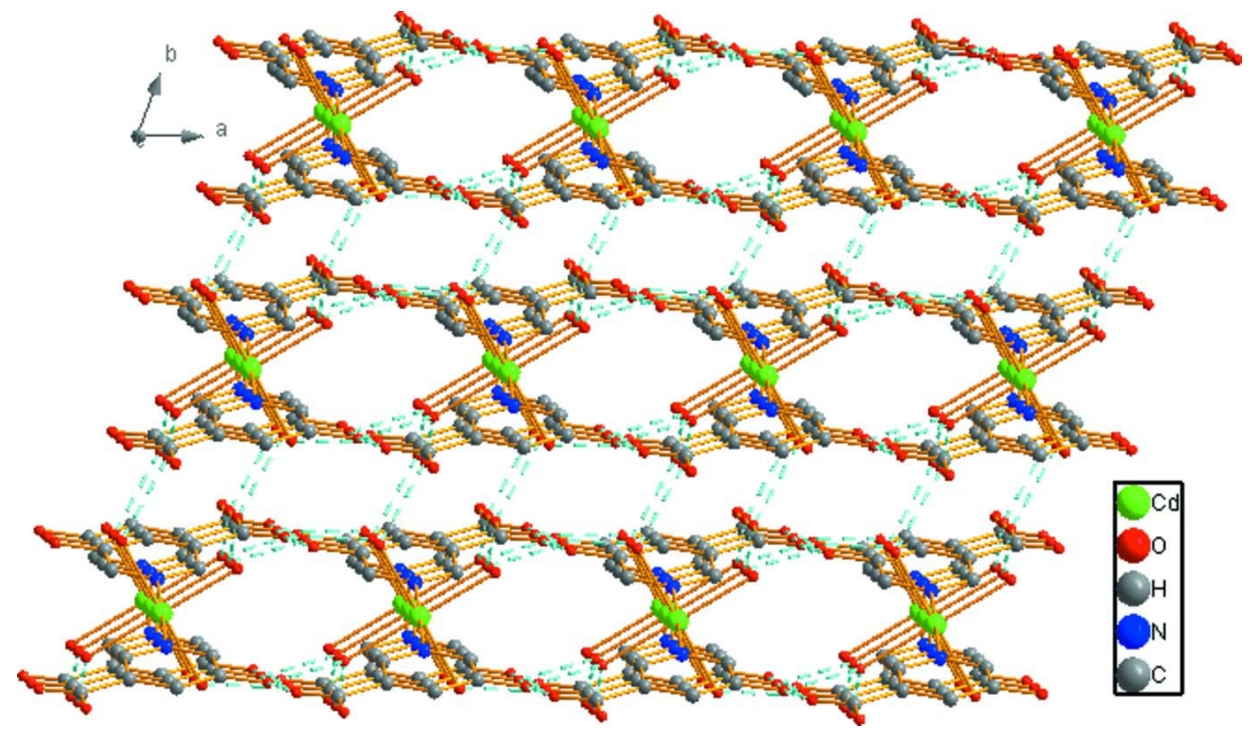

\section{Figure 2}

A view of the three dimensional framework of the title compound. The $\mathrm{O}-\mathrm{H} \cdots \mathrm{O}$ interactions are depicted by dashed lines.

\section{Tetraaquabis(5-hydroxynicotinato- $\kappa N)$ cadmium(II)}

Crystal data

$\left[\mathrm{Cd}\left(\mathrm{C}_{6} \mathrm{H}_{4} \mathrm{NO}_{3}\right)_{2}\left(\mathrm{H}_{2} \mathrm{O}\right)_{4}\right]$

$M_{r}=460.68$

Triclinic, $P \overline{1}$

Hall symbol: -P 1

$a=7.2190(1) \AA$

$b=7.2510(1) \AA$

$$
\begin{aligned}
& c=8.9260(1) \AA \\
& \alpha=70.377(1)^{\circ} \\
& \beta=68.154(1)^{\circ} \\
& \gamma=65.717(1)^{\circ} \\
& V=385.97(1) \AA^{3} \\
& Z=1
\end{aligned}
$$


$F(000)=230$

$D_{\mathrm{x}}=1.982 \mathrm{Mg} \mathrm{m}^{-3}$

Mo $K \alpha$ radiation, $\lambda=0.71073 \AA$

Cell parameters from 5615 reflections

$\theta=2.5-27.5^{\circ}$

\section{Data collection}

Bruker APEXII

diffractometer

Radiation source: fine-focus sealed tube

Graphite monochromator

$\omega$ scans

Absorption correction: multi-scan

(SADABS; Sheldrick, 1996)

$T_{\min }=0.667, T_{\max }=0.903$

\section{Refinement}

Refinement on $F^{2}$

Least-squares matrix: full

$R\left[F^{2}>2 \sigma\left(F^{2}\right)\right]=0.016$

$w R\left(F^{2}\right)=0.042$

$S=1.09$

1759 reflections

131 parameters

7 restraints

Primary atom site location: structure-invariant direct methods $\mu=1.48 \mathrm{~mm}^{-1}$

$T=296 \mathrm{~K}$

Sheet, colourless

$0.27 \times 0.17 \times 0.07 \mathrm{~mm}$

6067 measured reflections

1759 independent reflections

1754 reflections with $I>2 \sigma(I)$

$R_{\text {int }}=0.017$

$\theta_{\text {max }}=27.5^{\circ}, \theta_{\text {min }}=2.5^{\circ}$

$h=-9 \rightarrow 9$

$k=-9 \rightarrow 9$

$l=-11 \rightarrow 11$

Secondary atom site location: difference Fourier map

Hydrogen site location: inferred from neighbouring sites

$\mathrm{H}$ atoms treated by a mixture of independent and constrained refinement

$w=1 /\left[\sigma^{2}\left(F_{\mathrm{o}}^{2}\right)+(0.0249 P)^{2}+0.1253 P\right]$ where $P=\left(F_{\mathrm{o}}^{2}+2 F_{\mathrm{c}}^{2}\right) / 3$

$(\Delta / \sigma)_{\max }<0.001$

$\Delta \rho_{\max }=0.36 \mathrm{e} \AA^{-3}$

$\Delta \rho_{\min }=-0.34$ e $\AA^{-3}$

\section{Special details}

Geometry. All e.s.d.'s (except the e.s.d. in the dihedral angle between two 1.s. planes) are estimated using the full covariance matrix. The cell e.s.d.'s are taken into account individually in the estimation of e.s.d.'s in distances, angles and torsion angles; correlations between e.s.d.'s in cell parameters are only used when they are defined by crystal symmetry. An approximate (isotropic) treatment of cell e.s.d.'s is used for estimating e.s.d.'s involving l.s. planes.

Refinement. Refinement of $F^{2}$ against ALL reflections. The weighted $R$-factor $w R$ and goodness of fit $S$ are based on $F^{2}$, conventional $R$-factors $R$ are based on $F$, with $F$ set to zero for negative $F^{2}$. The threshold expression of $F^{2}>\sigma\left(F^{2}\right)$ is used only for calculating $R$-factors $(\mathrm{gt})$ etc. and is not relevant to the choice of reflections for refinement. $R$-factors based on $F^{2}$ are statistically about twice as large as those based on $F$, and $R$-factors based on ALL data will be even larger.

Fractional atomic coordinates and isotropic or equivalent isotropic displacement parameters $\left(\AA^{2}\right)$

\begin{tabular}{lllll}
\hline & $x$ & $y$ & $z$ & $U_{\text {iso }} * U_{\text {eq }}$ \\
\hline Cd1 & 0.5000 & 0.5000 & 1.0000 & $0.02626(6)$ \\
O1 & $0.8938(2)$ & $0.7990(2)$ & $0.32463(16)$ & $0.0427(3)$ \\
O1W & $0.2684(3)$ & $0.3168(3)$ & $1.07212(17)$ & $0.0482(4)$ \\
H1WA & $0.287(5)$ & $0.249(4)$ & $1.009(3)$ & $0.072^{*}$ \\
H1WB & $0.209(5)$ & $0.270(4)$ & $1.163(2)$ & $0.072^{*}$ \\
O2 & $0.6901(2)$ & $0.8709(2)$ & $0.16430(15)$ & $0.0429(3)$ \\
O2W & $0.7595(2)$ & $0.2074(2)$ & $0.92481(18)$ & $0.0478(4)$ \\
H2WA & $0.881(3)$ & $0.189(4)$ & $0.865(3)$ & $0.072^{*}$ \\
H2WB & $0.762(5)$ & $0.098(3)$ & $0.994(3)$ & $0.072^{*}$ \\
O3 & $0.0066(2)$ & $0.7675(2)$ & $0.58671(17)$ & $0.0400(3)$ \\
H3 & $0.002(4)$ & $0.785(4)$ & $0.491(2)$ & $0.053(7)^{*}$
\end{tabular}




\begin{tabular}{lllll}
$\mathrm{N} 1$ & $0.4459(2)$ & $0.6297(2)$ & $0.74305(16)$ & $0.0264(3)$ \\
$\mathrm{C} 1$ & $0.5945(2)$ & $0.6740(2)$ & $0.60554(19)$ & $0.0267(3)$ \\
$\mathrm{H} 1 \mathrm{~A}$ & 0.7280 & 0.6483 & 0.6138 & $0.032^{*}$ \\
$\mathrm{C} 2$ & $0.5560(2)$ & $0.7568(2)$ & $0.45137(18)$ & $0.0245(3)$ \\
$\mathrm{C} 3$ & $0.3595(3)$ & $0.7885(2)$ & $0.43778(19)$ & $0.0263(3)$ \\
$\mathrm{H} 3 \mathrm{~A}$ & 0.3310 & 0.8402 & 0.3354 & $0.032^{*}$ \\
$\mathrm{C} 4$ & $0.2061(2)$ & $0.7415(2)$ & $0.5802(2)$ & $0.0270(3)$ \\
$\mathrm{C} 5$ & $0.2549(2)$ & $0.6655(3)$ & $0.73035(19)$ & $0.0276(3)$ \\
$\mathrm{H} 5 \mathrm{~A}$ & 0.1507 & 0.6383 & 0.8260 & $0.033^{*}$ \\
$\mathrm{C} 6$ & $0.7273(3)$ & $0.8123(2)$ & $0.30121(19)$ & $0.0288(3)$ \\
\hline
\end{tabular}

Atomic displacement parameters $\left(\AA^{2}\right)$

\begin{tabular}{lllllll}
\hline & $U^{11}$ & $U^{22}$ & $U^{33}$ & $U^{12}$ & $U^{13}$ & $U^{23}$ \\
\hline Cd1 & $0.02499(9)$ & $0.03841(10)$ & $0.01604(8)$ & $-0.01493(7)$ & $-0.00552(6)$ & $-0.00099(6)$ \\
O1 & $0.0313(6)$ & $0.0688(9)$ & $0.0266(6)$ & $-0.0259(6)$ & $-0.0010(5)$ & $-0.0044(6)$ \\
O1W & $0.0589(9)$ & $0.0749(10)$ & $0.0276(6)$ & $-0.0484(8)$ & $-0.0017(6)$ & $-0.0091(7)$ \\
O2 & $0.0587(8)$ & $0.0592(8)$ & $0.0199(6)$ & $-0.0385(7)$ & $-0.0088(5)$ & $0.0028(5)$ \\
O2W & $0.0368(7)$ & $0.0424(7)$ & $0.0347(7)$ & $-0.0075(6)$ & $0.0072(6)$ & $0.0013(6)$ \\
O3 & $0.0285(6)$ & $0.0620(8)$ & $0.0326(7)$ & $-0.0220(6)$ & $-0.0135(5)$ & $0.0007(6)$ \\
N1 & $0.0258(6)$ & $0.0362(7)$ & $0.0187(6)$ & $-0.0141(5)$ & $-0.0068(5)$ & $-0.0018(5)$ \\
C1 & $0.0252(7)$ & $0.0376(8)$ & $0.0208(7)$ & $-0.0155(6)$ & $-0.0064(6)$ & $-0.0037(6)$ \\
C2 & $0.0278(7)$ & $0.0271(7)$ & $0.0195(7)$ & $-0.0128(6)$ & $-0.0046(6)$ & $-0.0033(5)$ \\
C3 & $0.0312(7)$ & $0.0288(7)$ & $0.0207(7)$ & $-0.0120(6)$ & $-0.0109(6)$ & $-0.0005(5)$ \\
C4 & $0.0249(7)$ & $0.0305(7)$ & $0.0281(7)$ & $-0.0112(6)$ & $-0.0107(6)$ & $-0.0026(6)$ \\
C5 & $0.0254(7)$ & $0.0358(8)$ & $0.0219(7)$ & $-0.0143(6)$ & $-0.0053(6)$ & $-0.0023(6)$ \\
C6 & $0.0340(8)$ & $0.0319(8)$ & $0.0206(7)$ & $-0.0166(6)$ & $-0.0025(6)$ & $-0.0038(6)$ \\
& & & & & &
\end{tabular}

Geometric parameters $\left(\AA,{ }^{\circ}\right)$

\begin{tabular}{llll}
\hline $\mathrm{Cd} 1-\mathrm{O} 2 \mathrm{~W}$ & $2.2830(14)$ & $\mathrm{O} 3-\mathrm{C} 4$ & $1.3543(19)$ \\
$\mathrm{Cd} 1-\mathrm{O} 2 \mathrm{~W}^{\mathrm{i}}$ & $2.2830(14)$ & $\mathrm{O} 3-\mathrm{H} 3$ & $0.830(17)$ \\
$\mathrm{Cd} 1-\mathrm{N} 1^{\mathrm{i}}$ & $2.2831(13)$ & $\mathrm{N} 1-\mathrm{C} 5$ & $1.335(2)$ \\
$\mathrm{Cd} 1-\mathrm{N} 1$ & $2.2831(13)$ & $\mathrm{N} 1-\mathrm{C} 1$ & $1.3411(19)$ \\
$\mathrm{Cd} 1-\mathrm{O} 1 \mathrm{~W}$ & $2.3291(13)$ & $\mathrm{C} 1-\mathrm{C} 2$ & $1.387(2)$ \\
$\mathrm{Cd} 1-\mathrm{O} 1 \mathrm{~W}^{\mathrm{i}}$ & $2.3291(13)$ & $\mathrm{C} 1-\mathrm{H} 1 \mathrm{~A}$ & 0.9300 \\
$\mathrm{O} 1-\mathrm{C} 6$ & $1.255(2)$ & $\mathrm{C} 2-\mathrm{C} 3$ & $1.385(2)$ \\
$\mathrm{O} 1 \mathrm{~W}-\mathrm{H} 1 \mathrm{WA}$ & $0.809(17)$ & $\mathrm{C} 2-\mathrm{C} 6$ & $1.517(2)$ \\
$\mathrm{O} 1 \mathrm{~W}-\mathrm{H} 1 \mathrm{WB}$ & $0.794(17)$ & $\mathrm{C} 3-\mathrm{C} 4$ & $1.389(2)$ \\
$\mathrm{O} 2-\mathrm{C} 6$ & $1.244(2)$ & $\mathrm{C} 3-\mathrm{H} 3 \mathrm{~A}$ & 0.9300 \\
$\mathrm{O} 2 \mathrm{~W}-\mathrm{H} 2 \mathrm{WA}$ & $0.823(17)$ & $\mathrm{C} 4-\mathrm{C} 5$ & $1.386(2)$ \\
$\mathrm{O} 2 \mathrm{~W}-\mathrm{H} 2 \mathrm{WB}$ & $0.822(17)$ & $\mathrm{C} 5-\mathrm{H} 5 \mathrm{~A}$ & 0.9300 \\
& & & $118.64(13)$ \\
$\mathrm{O} 2 \mathrm{~W}-\mathrm{Cd} 1-\mathrm{O} 2 \mathrm{~W}^{\mathrm{i}}$ & 180.0 & $\mathrm{C} 5-\mathrm{N} 1-\mathrm{C} 1$ & $117.86(10)$ \\
$\mathrm{O} 2 \mathrm{~W}-\mathrm{Cd} 1-\mathrm{N} 1^{\mathrm{i}}$ & $87.79(5)$ & $\mathrm{C} 5-\mathrm{N} 1-\mathrm{Cd} 1$ & $123.49(10)$ \\
$\mathrm{O} 2 \mathrm{~W}-\mathrm{Cd} 1-\mathrm{N} 1^{\mathrm{i}}$ & $92.21(5)$ & $\mathrm{C} 1-\mathrm{N} 1-\mathrm{Cd} 1$ & $122.29(14)$ \\
$\mathrm{O} 2 \mathrm{~W}-\mathrm{Cd} 1-\mathrm{N} 1$ & $92.21(5)$ & $\mathrm{N} 1-\mathrm{C} 1-\mathrm{C} 2$ & 118.9 \\
$\mathrm{O} 2 \mathrm{~W}-\mathrm{Cd} 1-\mathrm{N} 1$ & $87.79(5)$ & $\mathrm{N} 1-\mathrm{C} 1-\mathrm{H} 1 \mathrm{~A}$ &
\end{tabular}




\begin{tabular}{|c|c|c|c|}
\hline $\mathrm{N} 1{ }^{\mathrm{i}}-\mathrm{Cd} 1-\mathrm{N} 1$ & $180.000(1)$ & $\mathrm{C} 2-\mathrm{C} 1-\mathrm{H} 1 \mathrm{~A}$ & 118.9 \\
\hline $\mathrm{O} 2 \mathrm{~W}-\mathrm{Cd} 1-\mathrm{O} 1 \mathrm{~W}$ & $85.72(6)$ & $\mathrm{C} 3-\mathrm{C} 2-\mathrm{C} 1$ & $118.97(14)$ \\
\hline $\mathrm{O} 2 \mathrm{~W}^{\mathrm{i}}-\mathrm{Cd} 1-\mathrm{O} 1 \mathrm{~W}$ & $94.28(6)$ & $\mathrm{C} 3-\mathrm{C} 2-\mathrm{C} 6$ & $121.06(14)$ \\
\hline $\mathrm{N} 1{ }^{\mathrm{i}}-\mathrm{Cd} 1-\mathrm{O} 1 \mathrm{~W}$ & $90.57(5)$ & $\mathrm{C} 1-\mathrm{C} 2-\mathrm{C} 6$ & $119.96(14)$ \\
\hline $\mathrm{N} 1-\mathrm{Cd} 1-\mathrm{O} 1 \mathrm{~W}$ & $89.43(5)$ & $\mathrm{C} 2-\mathrm{C} 3-\mathrm{C} 4$ & $118.68(14)$ \\
\hline $\mathrm{O} 2 \mathrm{~W}-\mathrm{Cd} 1-\mathrm{O} 1 \mathrm{~W}^{\mathrm{i}}$ & $94.28(6)$ & $\mathrm{C} 2-\mathrm{C} 3-\mathrm{H} 3 \mathrm{~A}$ & 120.7 \\
\hline $\mathrm{O} 2 \mathrm{~W}^{\mathrm{i}}-\mathrm{Cd} 1-\mathrm{O} 1 \mathrm{~W}^{\mathrm{i}}$ & $85.72(6)$ & $\mathrm{C} 4-\mathrm{C} 3-\mathrm{H} 3 \mathrm{~A}$ & 120.7 \\
\hline $\mathrm{N} 1{ }^{\mathrm{i}}-\mathrm{Cd} 1-\mathrm{O} 1 \mathrm{~W}^{\mathrm{i}}$ & $89.43(5)$ & $\mathrm{O} 3-\mathrm{C} 4-\mathrm{C} 5$ & $115.78(14)$ \\
\hline $\mathrm{N} 1-\mathrm{Cd} 1-\mathrm{O} 1 \mathrm{~W}^{\mathrm{i}}$ & $90.57(5)$ & $\mathrm{O} 3-\mathrm{C} 4-\mathrm{C} 3$ & $125.40(14)$ \\
\hline $\mathrm{O} 1 \mathrm{~W}-\mathrm{Cd} 1-\mathrm{O} 1 \mathrm{~W}^{\mathrm{i}}$ & 180.0 & $\mathrm{C} 5-\mathrm{C} 4-\mathrm{C} 3$ & $118.81(14)$ \\
\hline $\mathrm{Cd} 1-\mathrm{O} 1 \mathrm{~W}-\mathrm{H} 1 \mathrm{WA}$ & $117(2)$ & $\mathrm{N} 1-\mathrm{C} 5-\mathrm{C} 4$ & $122.55(14)$ \\
\hline $\mathrm{Cd} 1-\mathrm{O} 1 \mathrm{~W}-\mathrm{H} 1 \mathrm{WB}$ & $127(2)$ & $\mathrm{N} 1-\mathrm{C} 5-\mathrm{H} 5 \mathrm{~A}$ & 118.7 \\
\hline $\mathrm{H} 1 \mathrm{WA}-\mathrm{O} 1 \mathrm{~W}-\mathrm{H} 1 \mathrm{WB}$ & $110(2)$ & $\mathrm{C} 4-\mathrm{C} 5-\mathrm{H} 5 \mathrm{~A}$ & 118.7 \\
\hline $\mathrm{Cd} 1-\mathrm{O} 2 \mathrm{~W}-\mathrm{H} 2 \mathrm{WA}$ & $131(2)$ & $\mathrm{O} 2-\mathrm{C} 6-\mathrm{O} 1$ & $125.02(15)$ \\
\hline $\mathrm{Cd} 1-\mathrm{O} 2 \mathrm{~W}-\mathrm{H} 2 \mathrm{WB}$ & $117(2)$ & $\mathrm{O} 2-\mathrm{C} 6-\mathrm{C} 2$ & $117.26(15)$ \\
\hline $\mathrm{H} 2 \mathrm{WA}-\mathrm{O} 2 \mathrm{~W}-\mathrm{H} 2 \mathrm{WB}$ & $105(2)$ & $\mathrm{O} 1-\mathrm{C} 6-\mathrm{C} 2$ & $117.71(14)$ \\
\hline $\mathrm{C} 4-\mathrm{O} 3-\mathrm{H} 3$ & $108.2(19)$ & & \\
\hline $\mathrm{O} 2 \mathrm{~W}-\mathrm{Cd} 1-\mathrm{N} 1-\mathrm{C} 5$ & $119.15(12)$ & $\mathrm{C} 1-\mathrm{C} 2-\mathrm{C} 3-\mathrm{C} 4$ & $1.8(2)$ \\
\hline $\mathrm{O} 2 \mathrm{~W}^{\mathrm{i}}-\mathrm{Cd} 1-\mathrm{N} 1-\mathrm{C} 5$ & $-60.85(12)$ & $\mathrm{C} 6-\mathrm{C} 2-\mathrm{C} 3-\mathrm{C} 4$ & $-177.83(14)$ \\
\hline $\mathrm{O} 1 \mathrm{~W}-\mathrm{Cd} 1-\mathrm{N} 1-\mathrm{C} 5$ & $33.45(13)$ & $\mathrm{C} 2-\mathrm{C} 3-\mathrm{C} 4-\mathrm{O} 3$ & $178.81(15)$ \\
\hline $\mathrm{O} 1 \mathrm{~W}^{\mathrm{i}}-\mathrm{Cd} 1-\mathrm{N} 1-\mathrm{C} 5$ & $-146.55(13)$ & $\mathrm{C} 2-\mathrm{C} 3-\mathrm{C} 4-\mathrm{C} 5$ & $0.2(2)$ \\
\hline $\mathrm{O} 2 \mathrm{~W}-\mathrm{Cd} 1-\mathrm{N} 1-\mathrm{C} 1$ & $-61.78(13)$ & $\mathrm{C} 1-\mathrm{N} 1-\mathrm{C} 5-\mathrm{C} 4$ & $1.7(2)$ \\
\hline $\mathrm{O} 2 \mathrm{~W}^{\mathrm{i}}-\mathrm{Cd} 1-\mathrm{N} 1-\mathrm{C} 1$ & $118.22(13)$ & $\mathrm{Cd} 1-\mathrm{N} 1-\mathrm{C} 5-\mathrm{C} 4$ & $-179.16(12)$ \\
\hline $\mathrm{O} 1 \mathrm{~W}-\mathrm{Cd} 1-\mathrm{N} 1-\mathrm{C} 1$ & $-147.47(13)$ & $\mathrm{O} 3-\mathrm{C} 4-\mathrm{C} 5-\mathrm{N} 1$ & $179.22(15)$ \\
\hline $\mathrm{O} 1 \mathrm{~W}^{\mathrm{i}}-\mathrm{Cd} 1-\mathrm{N} 1-\mathrm{C} 1$ & $32.53(13)$ & $\mathrm{C} 3-\mathrm{C} 4-\mathrm{C} 5-\mathrm{N} 1$ & $-2.1(2)$ \\
\hline $\mathrm{C} 5-\mathrm{N} 1-\mathrm{C} 1-\mathrm{C} 2$ & $0.5(2)$ & $\mathrm{C} 3-\mathrm{C} 2-\mathrm{C} 6-\mathrm{O} 2$ & $-5.8(2)$ \\
\hline $\mathrm{Cd} 1-\mathrm{N} 1-\mathrm{C} 1-\mathrm{C} 2$ & $-178.62(11)$ & $\mathrm{C} 1-\mathrm{C} 2-\mathrm{C} 6-\mathrm{O} 2$ & $174.61(15)$ \\
\hline $\mathrm{N} 1-\mathrm{C} 1-\mathrm{C} 2-\mathrm{C} 3$ & $-2.2(2)$ & $\mathrm{C} 3-\mathrm{C} 2-\mathrm{C} 6-\mathrm{O} 1$ & $173.06(16)$ \\
\hline $\mathrm{N} 1-\mathrm{C} 1-\mathrm{C} 2-\mathrm{C} 6$ & $177.42(14)$ & $\mathrm{C} 1-\mathrm{C} 2-\mathrm{C} 6-\mathrm{O} 1$ & $-6.6(2)$ \\
\hline
\end{tabular}

Symmetry code: (i) $-x+1,-y+1,-z+2$.

Hydrogen-bond geometry $\left(A,{ }^{\circ}\right)$

\begin{tabular}{lllll}
\hline$D-\mathrm{H} \cdots A$ & $D-\mathrm{H}$ & $\mathrm{H} \cdots A$ & $D \cdots A$ & $D-\mathrm{H} \cdots A$ \\
\hline $\mathrm{O} 1 W-\mathrm{H} 1 W A \cdots \mathrm{O} 2^{\mathrm{ii}}$ & $0.81(2)$ & $1.94(2)$ & $2.742(2)$ & $171(3)$ \\
$\mathrm{O} 1 W-\mathrm{H} 1 W B \cdots \mathrm{O} 3^{\text {iii }}$ & $0.79(2)$ & $2.20(2)$ & $2.9728(19)$ & $164(3)$ \\
$\mathrm{O} 2 W-\mathrm{H} 2 W A \cdots \mathrm{O} 1^{\text {iv }}$ & $0.82(2)$ & $1.87(2)$ & $2.6556(18)$ & $160(3)$ \\
$\mathrm{O} 2 W-\mathrm{H} 2 W B \cdots 2^{\mathrm{v}}$ & $0.82(2)$ & $1.93(2)$ & $2.7349(19)$ & $165(3)$ \\
$\mathrm{O} 3-\mathrm{H} 3 \cdots \mathrm{O} 1^{\mathrm{vi}}$ & $0.83(2)$ & $1.88(2)$ & $2.6637(19)$ & $157(3)$ \\
\hline
\end{tabular}

Symmetry codes: (ii) $-x+1,-y+1,-z+1$; (iii) $-x,-y+1,-z+2$; (iv) $-x+2,-y+1,-z+1$; (v) $x, y-1, z+1$; (vi) $x-1, y, z$. 